\title{
Accuracy of Conventional Triplane Measures Compared to 3-D Analysis for Assessment of Cubitus Varus Deformities in Adults
}

This article was published in the following Dove Press journal:

Therapeutics and Clinical Risk Management

\section{Gil-Sung Yoon \\ Won-Taek Oh (D) \\ Yong-Min Chun (D) \\ II-Hyun Koh (D) \\ Ho-Jung Kang \\ Yun-Rak Choi $\mathbb{D}$}

Department of Orthopedic Surgery, Severance Hospital, Yonsei University College of Medicine, Seoul 03722, South Korea
Correspondence: Yun-Rak Choi Department of Orthopedic Surgery, Yonsei University College of Medicine, 50-I Yonsei-ro, Seodaemun-gu, Seoul 03722, Republic of Korea

Tel +82-2-2228-2183

Fax +82-2-363-1139

Email YRCHOI@yuhs.ac
Purpose: Cubitus varus is a common triplane deformity in adults associated with supracondylar humeral fractures experienced as a child and consists of varus, extension, and internal rotation components. When corrective osteotomy is indicated, these three components should be measured precisely. This study aimed to evaluate the accuracy of radiographic and physical measurements of cubitus varus deformities in adults compared to values measured on three-dimensional (3-D) bone surface models of the adult bilateral humerus.

Methods: Three-dimensional bilateral humerus models were developed using bilateral humerus CT images of 20 adult patients with cubitus varus. The varus, internal rotation, and extension components of the deformity were assessed by superimposing the 3-D bone model onto a mirrorimage model of the contralateral normal humerus. Values obtained from the radiographic and physical measurements were compared with those from the 3D model. The reliability of each measurement was assessed by calculating correlation coefficients (CCs).

Results: Radiographic measurements of the varus and extension components showed good reliability ( $\mathrm{CC}=0.796$ and 0.791 , respectively). Physical measurement of the varus component, however, showed only moderate reliability $(\mathrm{CC}=0.539)$, while physical measurement of the extension and internal rotation components exhibited poor reliability $(\mathrm{CC}=0.164$ and 0.466 , respectively).

Conclusion: Varus and extension components of cubitus varus in adults can be reliably measured using conventional methods, whereas the internal rotation component cannot. Thus, 3-D methods with which to quantify the rotational component preoperatively might be needed when the correction of a rotational deformity is considered.

Keywords: cubitus varus, preoperative evaluation, triplane measurement, computed tomography

\section{Introduction}

Cubitus varus is a common deformity in adults associated with supracondylar humeral fractures experienced as a child. ${ }^{1-3}$ Although varus angulation is the most prominent component of this three-dimensional (3-D) deformity, ${ }^{4}$ extension and internal rotation of the distal humerus commonly exist. ${ }^{5-8}$ Although an unsightly appearance is often the main complaint of the deformity, long-lasting deformity can cause chronic joint pain, tardy ulnar nerve palsy, and posterolateral rotatory instability, even though these complaints are uncommon because surgical correction is usually performed in childhood. ${ }^{9-12}$ In adulthood, various osteotomy techniques, such as a simple lateral closing wedge, ${ }^{13}$ medial opening wedge, ${ }^{14}$ step-cut, ${ }^{15}$ 
pentalateral, ${ }^{7}$ dome,,${ }^{16-18}$ and 3-D osteotomy, ${ }^{5,8,19}$ have been recommended for correction of cubitus varus deformity.

Using optimal surgical technique, all three components (varus, extension, and internal rotation) of cubitus varus should be addressed to render satisfactory outcomes. A residual rotational deformity after corrective osteotomy not only could cause an unsatisfactory appearance after surgery, ${ }^{5,7,8,20}$ but could also be associated with tardy ulnar nerve palsy, ${ }^{10,11}$ pathologic elbow motion and muscle activity, ${ }^{8}$ and posterior instability of the ipsilateral shoulder. ${ }^{21}$ Accordingly, the accuracy of preoperative measures of the deformity can determine the degree of correction and clinical outcomes after surgical correction. Conventionally, the degrees of 3-D correction have been determined by measuring the components by physical and radiographic examinations in comparison to those of the contralateral side. $^{22,23}$ Meanwhile, advances in 3-D reconstruction and modeling software have allowed clinicians to accurately generate, process, and analyze 3-D surface models from 2-D medical imaging data. In 2011, Takeyasu et al analyzed the 3-D patterns of cubitus varus deformities and reported that only radiographic measurements of the varus component and physical measurements of the extension component were reasonably accurate, while radiographic measurements of the extension component and physical measurements of the internal rotation component were relatively inaccurate. ${ }^{23}$ However, the authors analyzed data from pediatric patients without skeletal maturity because cubitus varus deformities in adults are uncommon. Nonetheless, the 3-D patterns of cubitus varus deformity in adults could differ from those seen in children: secondary changes, such as osteoarthritis or posterolateral rotatory instability, to long-standing cubitus varus deformity are relatively common in clinical practice. ${ }^{24-26}$ Therefore, the accuracy of the conventional radiographic and physical methods evaluating the 3-D components of cubitus varus is unclear in adults.

We hypothesized that true 3-D humerus models of adult cubitus varus deformities can be obtained using customized software from high-resolution computed tomography (CT) data. Herein, the three components (varus, extension, and internal rotation) of cubitus varus were measured by comparison with mirrored contralateral normal 3-D humerus models. The purpose of this study was to evaluate the accuracy of conventional radiographic and physical measurements of the components in comparison with those measured on 3-D humerus models.

\section{Methods}

\section{Participants}

Between January 2010 and May 2013, 15 male and 5 female patients with unilateral cubitus varus deformities caused by malunion after distal humeral supracondylar fracture when they were children were enrolled in this study. The mean age was 35.4 years (range, 20-49 years), and the mean interval between original injury and image acquisition was 28.3 years (range, 16-44 years). The mean age at the time of injury was 7.1 years (range, 4-12 years). Original fractures were treated conservatively in all patients except two who underwent percutaneous pinning. One of the patients had posterolateral rotatory instability, and three patients had tardy ulnar nerve palsy.

\section{Three-Dimensional Evaluation of Cubitus Varus}

CT images of the affected and contralateral normal elbow (including the upper arm and forearm) with a $1.0-\mathrm{mm}$ slice thickness were obtained with a high-resolution CT scanner (SOMATOM sensation; SIEMENS, Germany). Digital imaging and communications in medicine (DICOM) data were imported to customized software (Mimics 14.01 software, Materialise, Leuven, Belgium), and 3D models of the affected and contralateral normal humeri were constructed (Figure 1A).

To evaluate humerus deformity in three dimensions, the affected humerus was compared with the mirror image of the contralateral normal humerus. In the distal humerus, the centers of the capitellum and trochlea were obtained with the use of a circle-fit algorithm at the lateral surface of the capitellum and the narrowest part of the trochlear groove, and the center (flexion-extension) axis was created as a line through the geometric centers of the trochlea and capitellum (Figure 1B). ${ }^{22}$ Then, the distal part of the model of the affected humerus was superimposed onto the corresponding part of the mirror image of the normal humerus to measure the degrees of varus and extension components of the deformity by point and surface registration using the medial and lateral epicondyles, the distal articular surface, and the flexion-extension axis as references (Figure 1C).

We quantified the varus component of the deformity by measuring the angle between the longitudinal axes of the affected and normal humeri on the true anteroposterior view (Figure 2A). The extension component was quantified using the same method on the true lateral view (Figure 2B). The 


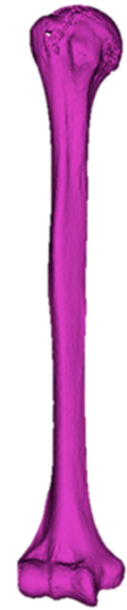

A
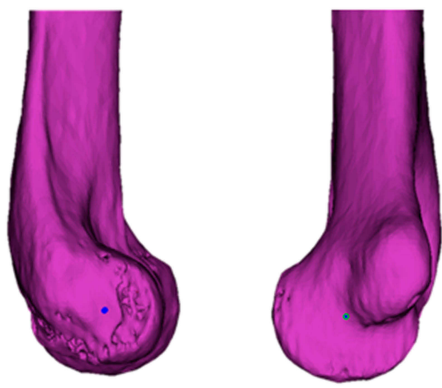

B

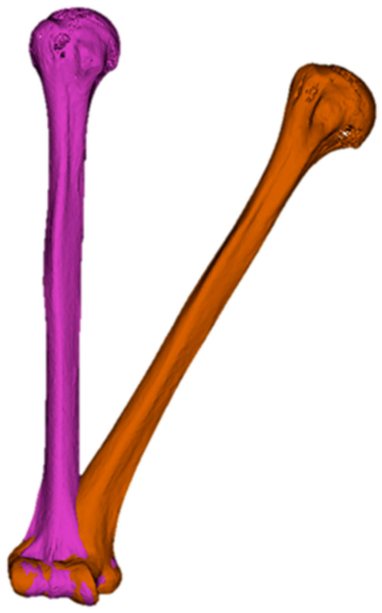

C

Figure I Using customized software (Mimics 14.0I software, Materialise, Leuven, Belgium), 3-D models of the affected and contralateral normal humeri were constructed (A). On the affected humerus and the mirror image of the contralateral normal humerus, the centers of the capitellum and trochlea were obtained with the use of a circle-fit algorithm at the lateral surface of the capitellum, and the center (flexion-extension) axis was created as a line through the geometric centers of the trochlea and capitellum (B). Then, the distal part of the model of the affected humerus was superimposed onto the corresponding part of the mirror image of the normal humerus to measure the degrees of varus and extension components of the deformity by point and surface registration using the medial and lateral epicondyles, the distal articular surface, and the flexion-extension axis as references $(\mathbf{C})$.

degree of internal rotation deformity was measured by comparing the retroversion angle of the affected humerus and that of the mirror image of the contralateral normal humerus. The retroversion angle was measured as the angle subtended by the flexion-extension axis of the distal humerus and the humeral head axis, which is the line between two points at $90^{\circ}$ to the anterior and the posterior articular margins (Figure 2C). ${ }^{27}$ To establish the interobserver reliability for these measurements, two experienced hand surgeons (IHK and YRC) evaluated all 3-D images, and the mean value of each measurement was used in this study.

\section{Radiographic Evaluation of Cubitus Varus}

For the anteroposterior view of the elbow, the forearm was positioned supine (palm up) on the radiographic table, with the

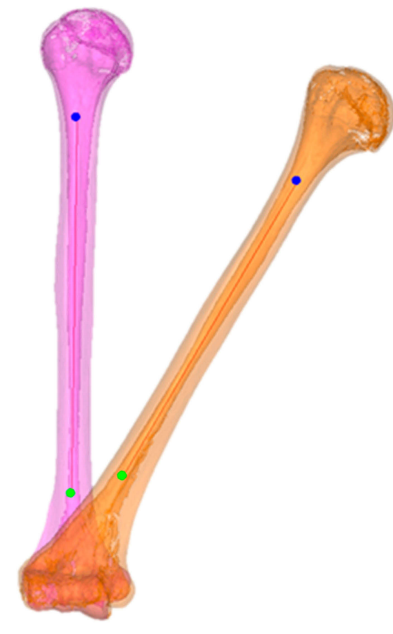

A

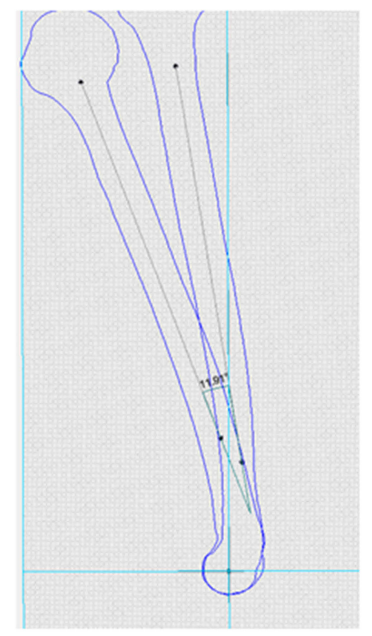

B

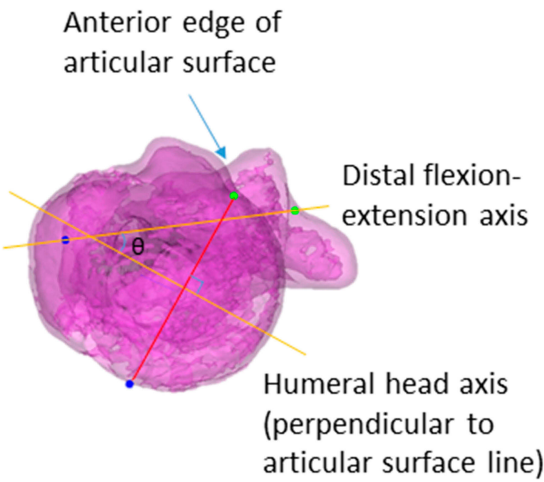

C

Figure 2 In three-dimensional measurements of cubitus varus, the anteroposterior view of the varus deformity angle (A) was obtained by measuring the angle between the humeral axes of the affected and normal humeri. Lateral view of the extension deformity angle $(\mathbf{B})$. The internal rotation deformity angle was measured as the difference in retroversion angles $(\theta)$ of the affected and normal humeri $(\mathbf{C})$. 
elbow joint fully extended. On the anteroposterior radiograph, the humerus-elbow-wrist angle (HEW-A) was defined by a line passing the longitudinal humeral axis and a line passing through the proximal and distal midpoints of the radius and ulna (Figure 3A). ${ }^{13,23}$ For the lateral view of the elbow, the forearm rested on its ulnar side on the radiographic cassette, with the joint flexed 90 degrees and the thumb positioned upward. On the lateral radiograph, the tilting angle (TA) was determined by the anterior tilt of the articular condyles with respect to the long axis of the humerus on a lateral radiograph (Figure 3B). ${ }^{23}$ To establish the interobserver reliability for the radiographic measurements, two experienced hand surgeons (IHK and YRC) evaluated all radiographs, and the mean value of each measurement was used in this study. Varus deformity angle and extension deformity angle were calculated as the differences in HEW-A and TA between the normal and affected sides, respectively.

\section{Physical Evaluation of Cubitus Varus}

We took photos of all patients at the time of physical examination to measure the carrying angle, the range of elbow flexion, and the internal rotation angle (IRA) of the humerus (Figure 3C-E). The extension deformity was determined by the difference in maximal elbow flexion by comparing the affected elbow with the contralateral one. ${ }^{19,21}$ The carrying angle was measured as the angle formed by the forearm and the humerus with elbow extension and forearm supination. For the measurement of elbow flexion, a goniometric axis was laterally placed and aligned with the lateral epicondyle of the humerus. The stationary arm was positioned parallel to the longitudinal axis of the humerus, pointing toward the tip of the acromial process, and the movable arm was positioned parallel to the longitudinal axis of the forearm, pointing toward the styloid process of the radius. The
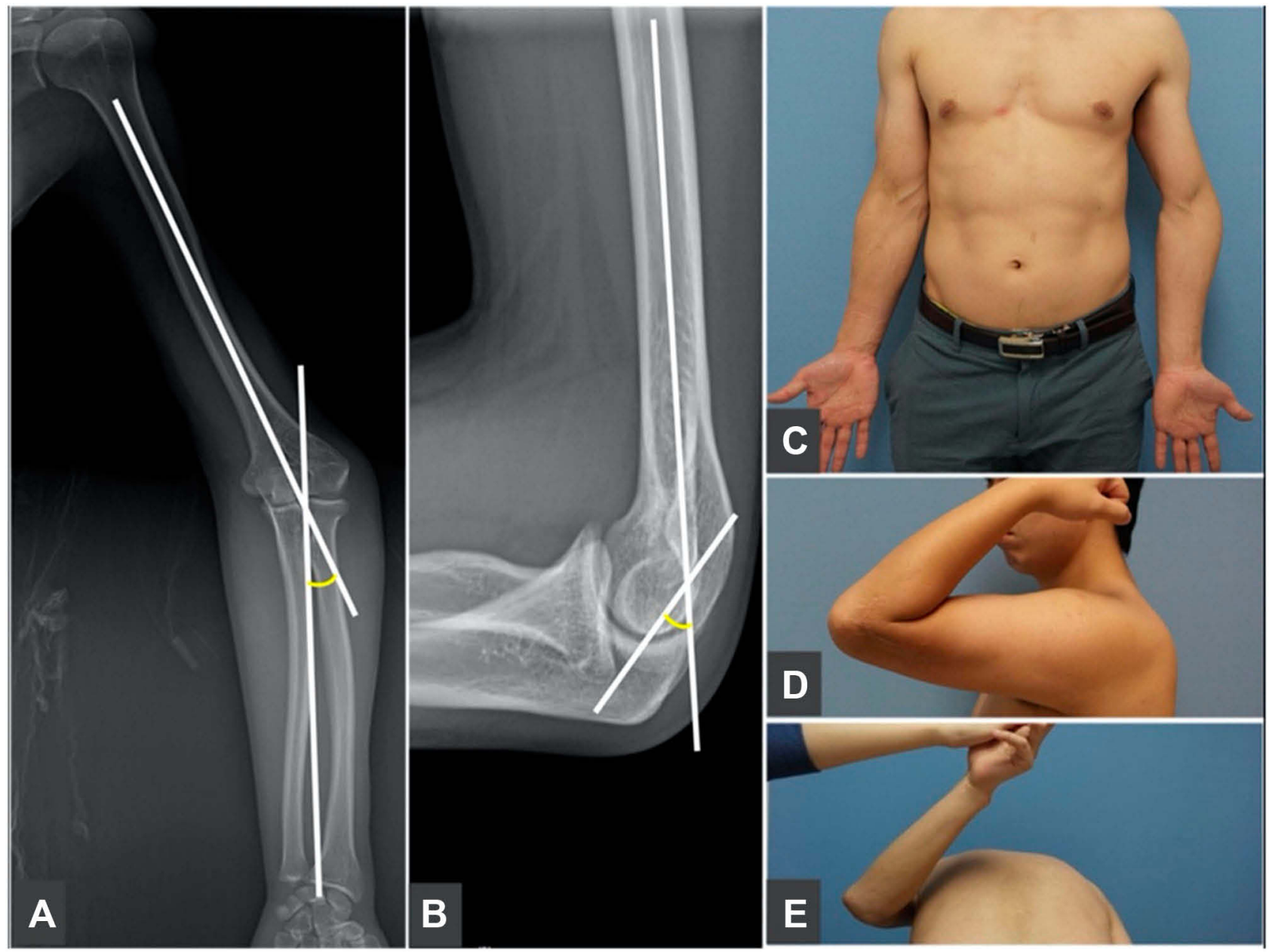

Figure 3 In radiographic measurements, the humerus-elbow-wrist angle (A) comprised the angle between the humeral axis and a line passing through the proximal and distal midpoints of the radius and ulna. The tilting angle (B) was determined by the anterior tilt of the articular condyles with respect to the humeral axis on a lateral radiograph. For physical measurements, the carrying angle (C), elbow flexion (D), and shoulder internal rotation (E) were estimated. 
IRA, obtained according to the method of Yamamoto et $\mathrm{al}^{20}$ was based on the difference in the rotational range of shoulder motion between the affected and normal sides. We defined the difference in IRA between the normal and affected sides as being the internal rotation deformity angle of the humerus. To establish the interobserver reliability for the physical evaluations, two experienced hand surgeons (IHK and YRC) evaluated each measurement, and the mean value was used in this study.

\section{Ethical Considerations}

Owing to the retrospective nature of this study, written consent from the involved patients was not obtained. Patient data confidentiality was maintained, and that this study was conducted in accordance with the Declaration of Helsinki. Our institutional review board approved the study and waived the requirement for informed consent (name of committee: Yonsei University Health System, Severance Hospital, Institutional Review Board; reference no. 4-2016-0103).

\section{Statistical Analyses}

We performed a Wilcoxon signed-rank test to compare the varus, extension, and internal rotation deformity angles among the three measurement methods. Intraclass correlation coefficients were used to assess the reliability of radiographic and physical measurements for varus, extension, and internal rotation deformity angles to 3-D measurements. The reliability was rated as "acceptable" if the correlation coefficient was 0.75 or greater. A $P$ value less than 0.05 was considered significant. Data were analyzed with MedCalc software, version 12.7 (MedCalc, Ostend, Belgium).

\section{Results}

The mean varus deformity angle was $29.4 \pm 5.6^{\circ}$ when measured according to carrying angle difference, $28.7 \pm$ $6.1^{\circ}$ when measured according to HEW-A difference, and $25.1 \pm 7.8^{\circ}$ when measured according to the 3 -D modeling method. When the 3-D measurement was considered accurate, intraclass correlation coefficients for the carrying angle and HEW-A methods for the 3-D measurement were 0.539 and 0.796 , respectively. Only the HEW-A method indicated good reliability (Table 1).

Mean extension deformity was $1.6 \pm 3.6^{\circ}$ when measured according to a difference in flexion, $10.2 \pm 12.1^{\circ}$ when measured according to a difference in TA, and $8.2 \pm 12.0^{\circ}$ when measured according to 3-D modeling. When the
Table I Comparison of Measurements for Each Component of Cubitus Varus

\begin{tabular}{|l|l|l|l|}
\hline \multirow{2}{*}{ Variable } & \multicolumn{3}{l|}{ Measurements } \\
\cline { 2 - 4 } & Three-Dimensional & Radiographic & Physical \\
\hline${\text { Deformity Angle }\left(^{\circ}\right)}$ & & & \\
Varus $^{\mathrm{a}}$ & $25.1 \pm 7.8$ & $28.7 \pm 6.1$ & $29.4 \pm 5.6$ \\
Extension $^{\mathrm{b}}$ & $8.2 \pm 12.0$ & $10.2 \pm 12.1$ & $1.6 \pm 3.6$ \\
Internal rotation $^{\mathrm{c}}$ & $12.5 \pm 11.7$ & & $8.5 \pm 12.8$ \\
\hline
\end{tabular}

Notes: Values are means \pm standard deviations. ${ }^{a}$ Varus deformity angle was assessed by humerus-elbow-wrist angle on radiographs and by carrying angle in physical measurement. ' Extension deformity angle was assessed by tilting angle on radiographs and by maximum flexion of the elbow in physical measurement. Internal rotation deformity angle was assessed by maximum internal rotation of the shoulder in physical measurement.

3-D measurement was considered accurate, intraclass correlation coefficients for a difference in flexion and a difference in TA for the 3-D measurement method were 0.164 and 0.791 , respectively. Only the TA method indicated good reliability.

Mean internal rotation deformity angle was $8.5 \pm 12.8^{\circ}$ when measured according to IRA and $12.5 \pm 11.7^{\circ}$ when measured according to the 3-D modeling method. When the 3-D measurement was considered accurate, intraclass correlation coefficient for the IRA method was 0.466 , indicating poor reliability.

\section{Discussion}

The purpose of this study was to evaluate the accuracy of conventional radiographic and physical measurements of three components of cubitus varus deformities in comparison to those from 3-D measurements using customized software from high-resolution CT data. Based on our results, only the varus component of cubitus varus could be measured accurately by physical and radiological evaluations; the extension component was measured accurately only by radiologic evaluation; and the internal rotation component of the deformity could not be measured accurately preoperatively using conventional methods.

This study had several limitations. First, we included a relatively small number of patients. Second, measurements of the three components of the cubitus varus deformity using 3D reconstruction models were believed to reflect a real cubitus varus deformity. According to a study by Takeyasu et al, ${ }^{23}$ the intra- and interobserver reliabilities for the 3D varus, extension, and internal rotation measurements were almost perfect with more than 0.90 of intraclass and interclass correlation coefficients. Despite these 
limitations, this is the first report on the 3D morphological analysis of the cubitus varus deformity in adult patients. We believe that the information obtained in this study will improve the understanding and surgical planning of the 3D deformity pattern in adult cubitus varus.

Several investigators have tried to analyze 3D components of the varus deformity using conventional radiography and physical examination in comparison with those of the contralateral arm. For the varus component, HEW-A radiography and the carrying angle on physical examination have been used. In this study, the mean varus deformity angle was approximately $29^{\circ}$ as measured by HEW-A, and a mean carrying angle difference of $4^{\circ}$ to the angle was measured by the 3D method. Measuring the varus component of the cubitus varus deformity in adults based on radiography, not on physical examination, showed acceptable reliability in this study. This finding suggests that preoperative planning using the varus component of the cubitus varus deformity in adults measured only by radiography seems to be acceptable to obtain true correction of the varus component of the deformity. This results are similar to the findings of Takeyasu et al in 2011 who analyzed 3D patterns of cubitus varus deformities in children mainly $(92 \%){ }^{23}$

The extension component of the cubitus varus deformity has been measured by comparing TA on lateral radiographs and the range of elbow movement of the affected side with that of the contralateral side. If patients with a cubitus varus deformity have limited elbow flexion due to extension malunion along with varus deformity, correction of the extension deformity is recommended along with varus correction. Our results showed that only the TA measurement had acceptable reliability and reflected the true extension deformity in adult patients with the cubitus varus deformity, which differs from the findings of Takeyasu et al who found that radiographic measurements of the extension component were inaccurate. ${ }^{23}$ These authors showed a relatively low interobserver reliability and that the radiographic measurement of the extension deformity (TA) was not always accurate in their patients. This difference might be because the subjects in our study were all adult cubitus varus patients. We believe that detection of reference points to measure TA is easier and more accurate in adult patients than in children. Therefore, surgeons should not correct an extension deformity based only on a difference in the range of elbow movement between the affected and contralateral elbows.

To quantify the internal rotational component of the cubitus varus deformity, Yamamoto et al proposed to use the difference in the internal rotation of shoulder motion. ${ }^{20}$ They reported that all of their patients had an internal rotational deformity of greater than $20^{\circ}$. The accuracy of the method for estimating the rotational component of the deformity proposed by Yamamoto et al is unclear, however. According to Takeyasu et $\mathrm{al}^{23}$ that method is relatively inaccurate, a finding that was also confirmed in our study. According to our study, measuring the internal rotation deformity using the difference in the internal rotation of the affected and contralateral shoulders showed no reliability in revealing the true internal rotational deformity of cubitus varus in adults. The limited range of the shoulder motion in addition to a bony malunion could affect the extent of passive internal rotation. Previously, Hindman et al proposed the use of axial humerus CT images to estimate the rotational deformity angle in patients with cubitus varus after supracondylar fracture. ${ }^{28}$ The use of CT images was also recommended to estimate the rotational deformity of the distal humerus preoperatively for accurate planning. ${ }^{23}$ Others have developed an operative method with the use of a custom-made surgical guide, designed on the basis of 3-D computer simulation with CT data, and have described its accuracy and usefulness for the treatment of cubitus varus deformity. ${ }^{29,30}$

\section{Conclusions}

The varus and extension components of cubitus varus appear to be measured accurately by HEW-A and TA measurements on plane radiographs, compared to physical measurements in adults. However, conventional methods to measure the rotational deformity component of the cubitus varus deformity in adults appear to be unreliable. Thus, if correction of the rotational component of the deformity is considered, CT images should be taken to quantify the rotational component of the deformity preoperatively.

\section{Acknowledgments}

The authors would like to thank Mr. Ha-Won Park for providing technical support in this study. The abstract of this paper was presented at the Congress in 2015 Federation of European Societies for Surgery of the Hand (FESSH) as a podium presentation. The podium's abstract was published in "FESSH Abstracts" in Journal of Hand Surgery (European Volume): https://doi.org/10.1177/1753193415579771.

\section{Disclosure}

The authors report no conflicts of interest in this work. 


\section{References}

1. O'Hara LJ, Barlow JW, Clarke NM. Displaced supracondylar fractures of the humerus in children. Audit changes practice. J Bone Joint Surg Br. 2000;82(2):204-210. doi:10.1302/0301-620X.82B2.9570

2. Weiland AJ, Meyer S, Tolo VT, Berg HL, Mueller J. Surgical treatment of displaced supracondylar fractures of the humerus in children. Analysis of fifty-two cases followed for five to fifteen years. J Bone Joint Surg Am. 1978;60(5):657-661. doi:10.2106/00004623-197860050-00012

3. Solfelt DA, Hill BW, Anderson CP, Cole PA. Supracondylar osteotomy for the treatment of cubitus varus in children: a systematic review. Bone Joint J. 2014;96-B(5):691-700. doi:10.1302/0301620X.96B5.32296

4. Chess DG, Leahey JL, Hyndman JC. Cubitus varus: significant factors. J Pediatr Orthop. 1994;14(2):190-192. doi:10.1097/01241398199403000-00011

5. Chung MS, Baek GH. Three-dimensional corrective osteotomy for cubitus varus in adults. $J$ Shoulder Elbow Surg. 2003;12(5):472-475. doi:10.1016/S1058-2746(03)00090-9

6. Kawanishi Y, Miyake J, Kataoka T, et al. Does cubitus varus cause morphologic and alignment changes in the elbow joint? J Shoulder Elbow Surg. 2013;22(7):915-923. doi:10.1016/j.jse.2013.01.024

7. Laupattarakasem W, Mahaisavariya B, Kowsuwon W, Saengnipanthkul S. Pentalateral osteotomy for cubitus varus. Clinical experiences of a new technique. J Bone Joint Surg Br. 1989;71 (4):667-670. doi:10.1302/0301-620X.71B4.2768319

8. Usui M, Miyano S, Narita H, Kura H. Three-dimensional corrective osteotomy for treatment of cubitus varus after supracondylar fracture of the humerus in children. J Shoulder Elbow Surg. 1995;4:17-22. doi:10.1016/S1058-2746(10)80003-5

9. Abe M, Ishizu T, Morikawa J. Posterolateral rotatory instability of the elbow after posttraumatic cubitus varus. J Shoulder Elbow Surg. 1997;6(4):405-409. doi:10.1016/S1058-2746(97)90011-2

10. Abe M, Ishizu T, Shirai H, Okamoto M, Onomura T. Tardy ulnar nerve palsy caused by cubitus varus deformity. $J$ Hand Surg Am. 1995;20(1):5-9. doi:10.1016/S0363-5023(05)80047-4

11. Mitsunari A, Muneshige H, Ikuta Y, Murakami T. Internal rotation deformity and tardy ulnar nerve palsy after supracondylar humeral fracture. J Shoulder Elbow Surg. 1995;4:23-29. doi:10.1016/S10582746(10)80004-7

12. O’Driscoll SW, Spinner RJ, McKee MD, et al. Tardy posterolateral rotatory instability of the elbow due to cubitus varus. $J$ Bone Joint Surg Am. 2001;83(9):1358-1369. doi:10.2106/00004623-20010900000011

13. Oppenheim WL, Clader TJ, Smith C, Bayer M. Supracondylar humeral osteotomy for traumatic childhood cubitus varus deformity. Clin Orthop Relat Res. 1984;188:34-39.

14. King D, Secor C. Bow elbow (cubitus varus). J Bone Joint Surg Am. 1951;33(3):572-576. doi:10.2106/00004623-195133030-00003

15. DeRosa GP, Graziano GP. A new osteotomy for cubitus varus. Clin Orthop Relat Res. 1988;236:160-165.

16. Kanaujia RR, Ikuta Y, Muneshige H, Higaki T, Shimogaki K. Dome osteotomy for cubitus varus in children. Acta Orthop Scand. 1988;59 (3):314-317. doi:10.3109/17453678809149371
17. Tien YC, Chih HW, Lin GT, Lin SY. Dome corrective osteotomy for cubitus varus deformity. Clin Orthop Relat Res. 2000;380:158-166. doi:10.1097/00003086-200011000-00021

18. Hahn SB, Choi YR, Kang HJ. Corrective dome osteotomy for cubitus varus and valgus in adults. $J$ Shoulder Elbow Surg. 2009;18 (1):38-43. doi:10.1016/j.jse.2008.07.013

19. Uchida Y, Ogata K, Sugioka Y. A new three-dimensional osteotomy for cubitus varus deformity after supracondylar fracture of the humerus in children. $J$ Pediatr Orthop. 1991;11(3):327-331. doi:10.1097/01241398-199105000-00009

20. Yamamoto I, Ishii S, Usui M, Ogino T, Kaneda K. Cubitus varus deformity following supracondylar fracture of the humerus. A method for measuring rotational deformity. Clin Orthop Relat Res. 1985;201:179-185.

21. Gurkan I, Bayrakci K, Tasbas B, Daglar B, Gunel U, Ucaner A. Posterior instability of the shoulder after supracondylar fractures recovered with cubitus varus deformity. J Pediatr Orthop. 2002;22 (2):198-202. doi:10.1097/01241398-200203000-00013

22. Brownhill JR, Furukawa K, Faber KJ, Johnson JA, King GJ. Surgeon accuracy in the selection of the flexion-extension axis of the elbow: an in vitro study. J Shoulder Elbow Surg. 2006;15(4):451-456. doi:10.1016/j.jse.2005.09.011

23. Takeyasu Y, Murase T, Miyake J, et al. Three-dimensional analysis of cubitus varus deformity after supracondylar fractures of the humerus. J Shoulder Elbow Surg. 2011;20(3):440-448. doi:10.1016/j.jse.2010. 11.020

24. Fujioka H, Nakabayashi Y, Hirata S, Go G, Nishi S, Mizuno K. Analysis of tardy ulnar nerve palsy associated with cubitus varus deformity after a supracondylar fracture of the humerus: a report of four cases. J Orthop Trauma. 1995;9:435e40. doi:10.1097/00005131199505000-00013

25. Ippolito E, Moneta MR, D'Arrigo C. Post-traumatic cubitus varus. Long-term follow-up of corrective supracondylar hu- meral osteotomy in children. J Bone Joint Surg Am. 1990;72:757e65. doi:10. 2106/00004623-199072050-00017

26. O'Driscoll SW, Bell DF, Morrey BF. Posterolateral rotatory instability of the elbow. J Bone Joint Surg Am. 1991;73:440e6. doi:10.2106/ 00004623-199173030-00015

27. Hill JA, Tkach L, Hendrix RW. A study of glenohumeral orientation in patients with anterior recurrent shoulder dislocations using computerized axial tomography. Orthop Rev. 1989;18:84-91.

28. Hindman BW, Schreiber RR, Wiss DA, Ghilarducci MJ, Avolio RE. Supracondylar fractures of the humerus: prediction of the cubitus varus deformity with CT. Radiology. 1988;168(2):513-515. doi:10. 1148/radiology.168.2.3393673

29. Omori S, Murase T, Oka K, et al. Postoperative accuracy analysis of three-dimensional corrective osteotomy for cubitus varus deformity with a custom-made surgical guide based on computer simulation. J Shoulder Elbow Surg. 2015;24:242e9. doi:10.1016/j.jse.2014.08.020

30. Takeyasu Y, Oka K, Miyake J, Kataoka T, Moritomo H, Murase T. Preoperative, computer simulation-based, three-dimensional corrective osteotomy for cubitus varus deformity with use of a custom-designed surgical device. J Bone Joint Surg Am. 2013;95: e173. doi:10.2106/JBJS.L.01622
Therapeutics and Clinical Risk Management

\section{Publish your work in this journal}

Therapeutics and Clinical Risk Management is an international, peerreviewed journal of clinical therapeutics and risk management, focusing on concise rapid reporting of clinical studies in all therapeutic areas outcomes, safety, and programs for the effective, safe, and sustained use of medicines. This journal is indexed on PubMed Central, CAS,
EMBase, Scopus and the Elsevier Bibliographic databases. The manuscript management system is completely online and includes a very quick and fair peer-review system, which is all easy to use. Visit http://www.dovepress.com/testimonials.php to read real quotes from published authors. 